\title{
Enhancement of anticancer efficacy using modified lipophilic nanoparticle drug encapsulation
}

This article was published in the following Dove Press journal:

International Journal of Nanomedicine

9 February 2012

Number of times this article has been viewed

Puiyan Lee'

Ruizhong Zhang'

Vincent $\mathrm{Li}^{\prime}$

Xuelai Liu'

Raymond WY Sun ${ }^{2}$

Chi-Ming Che ${ }^{2}$

Kenneth KY Wong'

'Department of Surgery, Li Ka Shing Faculty of Medicine, ${ }^{2}$ Department of Chemistry and Open Laboratory of Chemical Biology of the Institute of Molecular Technology for Drug Discovery and Synthesis, The University of Hong Kong, Hong Kong
Correspondence: Kenneth KY Wong Department of Surgery, The University of Hong Kong, Queen Mary Hospital, Pokfulam Road, Hong Kong

Tel +85222553486

Fax +85228173155

Email kkywong@hku.hk
Background: Development of anticancer drugs is challenging. Indeed, much research effort has been spent in the development of new drugs to improve clinical outcomes with minimal toxicity. We have previously reported that a formulation of lipid gold porphyrin nanoparticles reduced systemic drug toxicity when compared with free gold porphyrin. In this study, we investigated the delivery and treatment efficiency of PEG surface-modified lipid nanoparticles as a carrier platform.

Methods: We encapsulated antitumor drugs into PEG-modified lipid nanoparticles and these were characterized by size, zeta potential, and encapsulation efficiency. The delivery efficiency into tumor tissue was evaluated using a biodistribution study. To evaluate antitumor efficacy, gold porphyrin or camptothecin (a DNA topoisomerase I inhibitor) were encapsulated and compared using an in vivo neuroblastoma (N2A) model.

Results: We showed that drug encapsulation into PEG-modified lipid nanoparticles enhanced the preferential uptake in tumor tissue. Furthermore, higher tumor killing efficiency was observed in response to treatment with PEG-modified lipid nanoparticles encapsulating gold porphyrin or camptothecin when compared with free gold porphyrin or free camptothecin. The in vivo antitumor effect was further confirmed by study of tumor inhibition and positive apoptosis activity. Surface modification of lipophilic nanoparticles with PEG increased the efficiency of drug delivery into tumor tissue and subsequently more effective antitumor activity.

Conclusion: This specific design of a chemotherapeutic agent using nanotechnology is important in the development of a safe and effective drug in cancer therapy.

Keywords: lipid nanoparticles, gold porphyrin, cancer, neuroblastoma, camptothecin

\section{Introduction}

Chemotherapy is essential for the treatment of a variety of cancers. Although the use of modern anticancer drugs has improved the clinical outcome for patients with a wide range of cancers, the same could not be said for neuroblastoma, which is the most common solid tumor in childhood. ${ }^{1}$ This is because this tumor often presents with only few symptoms in the early clinical stages. Furthermore, patients often become refractory to available drugs very quickly. Thus, there is an urgent need for newer agents if we are to improve the overall survival of these patients.

It has been estimated that approximately one third of potent anticancer drugs are hydrophobic. A recently developed chemotherapeutic agent, gold (III) porphyrin (GP), is a water-insoluble inorganic metal complex and has been demonstrated to have efficacy against a wide range of cancers..$^{2-4}$ Indeed, its antitumor activity is 100 -fold more potent than cisplatin, an agent commonly used for treating neuroblastoma. ${ }^{2,3}$ 
Possible mechanisms of tumor killing include induction of apoptosis, the mitochondria-mediated apoptotic pathway, and production of reactive oxygen species. ${ }^{5}$ Similarly, another potent anticancer drug, camptothecin, features a closed hydrophobic lactone ring. ${ }^{6}$ Although the hydrophobicity of camptothecin results in more favorable antitumor activity, such as against hepatocellular, ovarian, breast, and renal tumors, ${ }^{7-10}$ its clinical application is limited because of severe systemic organ damage associated with significant side effects. ${ }^{6}$ Its in vivo toxicity has been reported to be correlated with low solubility and instability in the aqueous environment. ${ }^{11}$

Drug encapsulation via an optimal delivery system is an integral part of drug design to overcome the issues of systemic toxicity as well as to improve the efficiency of drug delivery. A number of studies have focused on the design of carriers for delivery to provide optimal incorporation of drugs. ${ }^{12-14}$ To the best of our knowledge, effective encapsulation of hydrophobic drugs into a carrier for the treatment of neuroblastoma has not yet been reported. With evolution in nanotechnology, the design of a drug delivery system with more controllable physical properties has shown promising results in enhancing antitumor activity in target tumor tissue. ${ }^{15}$ Tumor vasculature is characterized uniquely with leaky blood vessels, which allow nanoparticles greater than $100 \mathrm{~nm}$ in diameter to pass through. ${ }^{16}$ Thus, larger nanoparticles of 100-200 nm in diameter are ideal for delivery into tumor tissue and consequently can reduce renal clearance and uptake by the reticuloendothelial system. ${ }^{17}$ This phenomenon is called the "enhanced permeability retention" effect. ${ }^{15}$

For our delivery system, we used lipid nanoparticles consisting of cetyl alcohol and Brij 78 to incorporate GP, because they have been shown to have excellent biocompatibility in blood. ${ }^{18}$ The formulation strategy was to incorporate a microemulsion into a lipid nanoparticle carrier in distilled water as opposed to the organic solvents used in the synthesis of most other formulations. The solvent was carefully chosen in order to address the common problem of additional toxicity associated with use of an organic solvent. In our previous study, surface modification of lipid nanoparticles was shown to reduce the toxicity of GP, both in vitro and in vivo. ${ }^{19}$ In this work, we investigated the treatment efficiency of a lipophilic carrier by physical characterization, tumor accumulation, systemic distribution, and antitumor activity. To examine further the efficiency of a lipophilic carrier platform for delivery, we encapsulated camptothecin into the lipophilic carrier and investigated the activity and therapeutic effect of lipophilic-encapsulated camptothecin.

\section{Materials and methods Synthesis of PEGylated nanoparticles}

Gold (III) porphyrin 1a was manufactured and kindly provided by the Department of Chemistry at Hong Kong University. ${ }^{20}$ Camptothecin was purchased from Sigma-Aldrich (St Louis, $\mathrm{MO})$. The GP and camptothecin nanoparticles were prepared as previously described using an oil/water $(\mathrm{o} / \mathrm{w})$ microemulsion. ${ }^{19}$ Briefly, the drug was melted into the oil phase of cetyl alcohol at $60^{\circ} \mathrm{C}$. Brij 78, a surfactant, was added to the oil phase and stirred for 5 minutes. Methoxypoly(ethylene glycol) (mPEG 750-2000 Da, $5 \mathrm{~mol} \%$ ) was added to the nanoparticle template and mixed well by stirring. The particle size and surface charge of the nanoparticles was measured using a Zetasizer Nanoseries (Malvern, Westborough, MA). Then, $10 \mu \mathrm{L}$ of each sample was added to $1 \mathrm{~mL}$ of $1: 100(\mathrm{v} / \mathrm{v})$ phosphatebuffered saline ( $\mathrm{pH}$ 7.4) in distilled water for the zeta potential study, as previously described. ${ }^{21}$

\section{Encapsulation efficiency}

Drug entrapment efficiencies were determined by separating free GP from the GP nanoparticles (GPNP) using a Centrisart I centrifugal ultrafiltration unit (Supleco, Bellefonte, PA) with a filter membrane (molecular weight cutoff $10 \mathrm{kDa}$ ) as described elsewhere, ${ }^{22}$ and then measuring the GP in nanoparticle-containing supernatants using inductively coupled plasma-mass spectroscopy. About $1 \mathrm{~mL}$ of the formulation solution was put in the outside chamber and in the recovery chamber at the top. After separation by centrifugation at $4000 \mathrm{rpm}$ for 30 minutes, GPNP remained in the outer chamber while free GP was in the recovery chamber. To ensure mass balance, the filtrates were also assayed for GP. GP loading and entrapment efficiency were calculated as follows:

Percent drug entrapment efficiency $=($ GP entrapped in NP $)$ $/($ Total GP added into NP preparation $) \times 100 \%$

\section{Tumor cell lines and in vitro cytotoxicity test}

A murine glioblastoma (D54) or neuroblastoma (N2A) cell line was seeded in a 96-well plate at a density of $8 \times 10^{3}$ cells/well and cultured in Dulbecco's Modified Eagle Medium supplemented with 10\% fetal bovine serum and $1 \%$ penicillin/streptomycin at $37^{\circ} \mathrm{C}$. An MTT assay was performed after incubation of the lipophilic nanoparticleencapsulated drugs for 24 and 72 hours. Normal fibroblasts was seeded at a density of 8000 cells/well in a 96-well plate. The MTT assay was performed according to the 
manufacturer's protocol after incubation of the lipophilic nanoparticle-encapsulated drugs for 4 hours. Briefly, $10 \mu \mathrm{L}$ of MTT substrate was added into each well and incubated for 4 hours at $37^{\circ} \mathrm{C}$, after which $100 \mu \mathrm{L}$ of solubilization buffer was added to each well and incubated overnight. The absorbance measurement was performed at $540 \mathrm{~nm}$ with the reference filter at $650 \mathrm{~nm}$ using an absorbance microplate reader (BioRad, Hercules, CA).

\section{In vivo neuroblastoma model}

Six- to eight-week-old male A/J mice weighing $20 \pm 2 \mathrm{~g}$ were obtained and housed in the Laboratory Animal Unit, University of Hong Kong. The experimental protocol was approved by the Committee on the Use of Live Animals for Teaching and Research, University of Hong Kong (CULATR 1901-09). The animals were cared for humanely. N2A tumor-bearing mice were created by subcutaneous injection of $200 \mu \mathrm{L}$ of a mouse neuroblastoma cell line (N2A) at a density of $1.5 \times 10^{6} / \mathrm{mL}$ suspended in phosphate-buffered saline into the right flank of the mouse. At 8-11 days after inoculation, tumor mass became visible and measurable at the injection site. Tumor growth was evaluated by measuring the tumor volume using morphometric analysis with calipers every other day until 21 days.

Chemotherapy using 3-5 $\mathrm{mg} / \mathrm{kg}$ of free GP, GPNP, or GPNP surface-coated with PEG (GPNP-PEG) was administered intraperitoneally after the tumor became visible and the dose was chosen according to our previous report. ${ }^{19}$ Intraperitoneal administration was selected because this route has been demonstrated to be an effective and well tolerated systemic route as compared with intravenous administration to treat neuroblastoma. ${ }^{23-26}$ Tumor volume was calculated as height $\times$ weight $\times$ length. Body weight was measured every other day until 21 days. Percentage survival was determined by the number of mice surviving at the designated time points.

\section{Biodistribution study of gold porphyrin}

The liver, kidneys, and tumor tissue were harvested from each mouse, which was either untreated or had received $3 \mathrm{mg} / \mathrm{kg}(0.06 \mathrm{mg} / 20 \mathrm{~g})$ of free GP or GPNP conjugated with or without PEG at 6 and 24 hours. The harvested tissues were acid-digested as previously described. ${ }^{27}$ Biodistribution analysis was conducted by measuring the gold isotope concentration ( $\mathrm{ppb}$ ) with inductively coupled plasma-mass spectroscopy in the Bioengineering Department at the University of Science and Technology, Hong Kong.

\section{TUNEL assay}

A TUNEL assay was performed on $5 \mathrm{~mm}$ tumor paraffin sections using a commercially available kit (Roche Applied Science, Gilroy, CA) according to the manufacturer's instructions. Briefly, the tissue was fixed in $4 \%$ paraformaldehyde, dehydrated, paraffin-embedded, and sectioned. The section was then deparaffinized, rehydrated, and pretreated with $0.02 \mathrm{mg} / \mathrm{mL}$ of proteinase $\mathrm{K}$ in $0.1 \mathrm{mM}$ Tris-HCl pH8 buffer for 10 minutes. The TUNEL assay was performed by incubating fluorescein-conjugated terminal deoxynucleotidyl transferase in enzyme solution for 2 hours. The stained section was viewed using a fluorescent microscope under a blue filter.

\section{Statistical analysis}

The statistical analyses were performed using Student's paired $t$-test; a $P$ value $<0.05$ was considered to be statistically significant. The $\alpha$ value adjustment was made by Bonferroni correction for multiple comparisons. The data are presented as the mean \pm standard deviation.

\section{Results and discussion}

We synthesized lipophilic nanoparticles using cetyl alcohol and Brij 78 surfactant. This lipophilic formulation had been developed earlier in our laboratory to incorporate lipophilic GP, and we showed that GPNP had a significant reduction in side effects when compared with free GP in a neuroblastoma model. ${ }^{19}$ Nonetheless, prolonged administration of this early formulation still resulted in systemic toxicity due to accumulation in the liver.

In this study, we surface-coated GPNP with PEG to investigate if this modification could enhance drug delivery into tumor tissue and also reduce systemic uptake. The lipophilic nanoparticles with or without surface modification were first characterized (Table 1). More than $90 \%$ of hydrophobic GP was encapsulated into PEG-coated lipophilic nanoparticles. The zeta potential of GP, GPNP, and GPNP-PEG ranged from $-4.13 \mathrm{mV}$

Table I Biophysical characterization of lipophilic carrier formulations and free GP.The results are presented as the mean \pm standard deviation

\begin{tabular}{lccl}
\hline Formulations & $\begin{array}{l}\text { Particle size } \\
(\mathbf{n m})\end{array}$ & $\begin{array}{l}\text { Zeta potential } \\
(\mathbf{m V})\end{array}$ & $\begin{array}{l}\text { Encapsulation } \\
\text { efficiency }\end{array}$ \\
\hline GP & $10.91 \pm 6.02$ & $-12.49 \pm 6.03$ & \\
GPNP & $104.4 \pm 18.5$ & $-4.13 \pm 8.19$ & \\
GPNP-PEG & $164.47 \pm 6.9$ & $-8.5 \pm 2.72$ & $98 \%$ of GP \\
\hline
\end{tabular}

Abbreviations: GP, gold porphyrin; GPNP, gold porphyrin nanoparticles; GPNP-PEG, gold porphyrin nanoparticles surface-coated with PEG. 
to $-12.49 \mathrm{mV}$, suggesting a slight change in value but within a low negative range of net surface charge after PEG modification, possibly due to nanoparticle encapsulation. The low negative value of the surface charge would mean that the favorable binding affinity for cells remained unchanged after encapsulation. ${ }^{28}$ Direct light scattering measurements in Table 1 showed that encapsulation increased the size of GPNP compared with GP. The addition of PEG further increased the size of the lipophilic nanoparticles from $101.9 \mathrm{~nm}$ to $164.4 \mathrm{~nm}$ without a significant change in zeta potential or morphology. This would benefit delivery into tumor tissue via the enhanced permeability retention effect. Because the pore sizes of the liver fenestrae are around $100 \mathrm{~nm}$ and the gap sizes of the tumor vessels are approximately $400 \mathrm{~nm},{ }^{29-31}$ we hypothesized that GPNP-PEG could evade uptake by the liver, with enhanced tumor uptake. This is indeed shown in Figure 1. Here, accumulation of free GP in the liver was $336 \mathrm{ppb} \mathrm{Au} / \mathrm{g}$ tissue at 6 hours, and decreased to $112 \mathrm{ppb} \mathrm{Au/g}$ tissue at 24 hours, while both the kidney and tumor tissue showed minimal uptake $(<30 \mathrm{ppb} \mathrm{Au} / \mathrm{g}$ tissue) at 6 and 24 hours, respectively. The unconjugated GPNP showed comparable uptake in the liver and kidney (18 ppb Au/g tissue and $52 \mathrm{ppb} \mathrm{Au/g}$ tissue, respectively) and relatively high tumor uptake at 6 hours. At 24 hours, uptake of unconjugated GPNP by the liver increased but was not significantly different from that in tumor tissue. On the other hand, GPNP-PEG showed only minimal liver and kidney uptake (6-44 ppb Au/g tissue), whilst uptake in tumor tissue was significantly higher (468 ppb Au/g tissue and $392 \mathrm{ppb} \mathrm{Au/g}$ tissue, respectively, $P<0.05$ ) at 6 and 24 hours. This finding would explain the reduced systemic toxicity after encapsulation into a PEG surfacecoated lipophilic carrier.

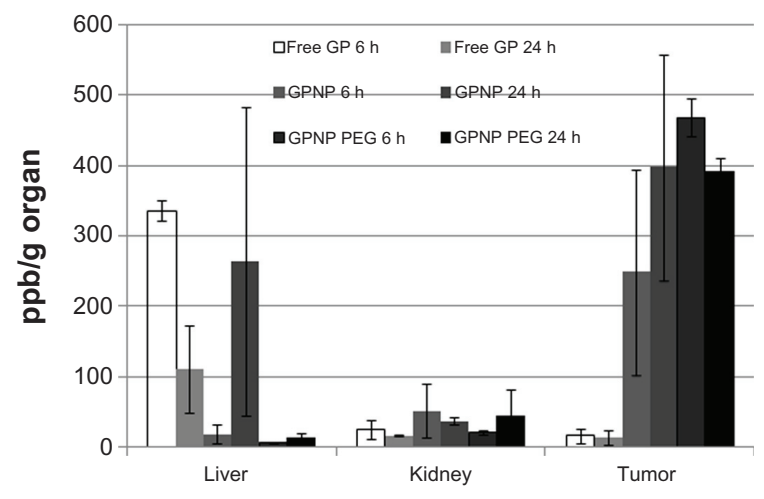

Figure I Tissue residence of free GP, GPNP, and GPNP-PEG at 6 and 24 hours. Notes: Data are presented as the $\mathrm{ppb} A \mathrm{Au} / \mathrm{g}$ tissue \pm standard deviation $(* P<0.05, \mathrm{n}=3$ ). Abbreviations: GP, gold porphyrin; GPNP, gold porphyrin nanoparticles; GPNP-PEG, gold porphyrin nanoparticles surface-coated with PEG.
Another requirement of a carrier system in cancer therapy is the ability to facilitate the antitumor activity of the active drug. Our in vitro study investigated the activity of the encapsulated drug against tumor cells. Here, MTT assay showed that greater cell death was seen in the N2A neuroblastoma cell line using encapsulated GP in a dose-dependent manner (Figure 2A).

This would suggest that the lipophilic carrier facilitated antitumor activity against N2A. In order to confirm if our carrier template could enhance antitumor activity of other drugs, we tested camptothecin, another potent hydrophobic drug, using the same carrier system, whereby the PEG-coated lipophilic carrier also enhanced antitumor activity. The $\mathrm{IC}_{50}$ of N2A for GPNP was $0.002 \mathrm{mM}$ as compared with $0.005 \mathrm{mM}$ of GP, and was $0.0025 \mathrm{mM}$ for camptothecin-NP or camptothecin-NP-PEG and $0.03 \mathrm{mM}$ for free camptothecin. This result indicated that lipophilic carrier enhanced drug efficacy by $2-10$-fold.

On the other hand, because insertion of long-chained PEG provided steric hindrance to prevent cellular and serum protein interaction, we investigated whether the composition

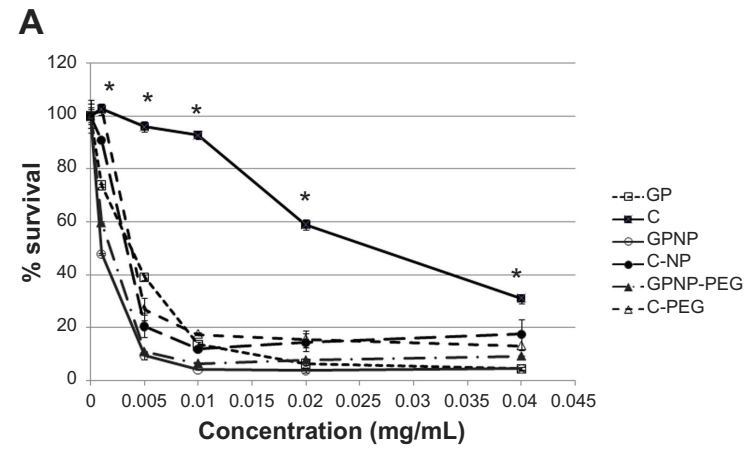

B

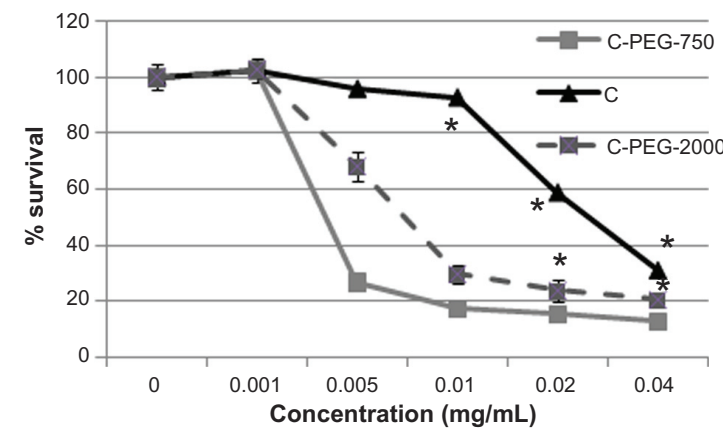

Figure 2 (A) Dose-dependent antitumor activity against N2 A for GP and camptothecin with or without the lipophilic carrier formulation $(n=4$, *P $<0.05$ when comparing nanoparticle formulation with free GP or camptothecin). (B) Comparison of surfacecoated lipophilic nanoparticle carrier with different molecular weights (750 Da and 2000 Da) of PEG.

Notes: Results are presented as the mean \pm standard deviation $(n=4, * P<0.05$ for C-PEG 750 or C-PEG 2000 in comparison with C).

Abbreviations: C, camptothecin; GP, gold porphyrin; GPNP, gold porphyrin nanoparticles; GPNP-PEG, gold porphyrin nanoparticles surface-coated with PEG. 
of PEG would interfere with the antitumor activity of the encapsulated drug. Indeed, less antitumor activity was found when the nanoparticle carrier was coated with higher molecular weight PEG (PEG 2000), as compared with lower molecular weight PEG (PEG 750), as shown in Figure 2B.

We next investigated the in vivo therapeutic effect of GPNP-PEG using a neuroblastoma model. As shown in Figure 3, GPNP-PEG doses of 3, 4 and $5 \mathrm{mg} / \mathrm{kg}$ all had a significant antitumor effect by inhibiting the growth of N2A cells when compared with the control $(P<0.05)$. The antitumor effect decreased after the first 7 days but could be sustained by repeated doses.

Because evasion of cell death is an indicator of drug resistance, ${ }^{32}$ we further studied tumor cell apoptosis using the TUNEL assay after treatment with the lipophilic nanoparticle carrier. Figure 4 demonstrates that apoptotic activity was significantly higher in the GPNP-PEG-treated neuroblastoma line than in the untreated neuroblastoma line. Similar findings was also recorded when camptothecin-PEG was given. Taken together, positive tumor inhibition as well as apoptosis indicates that our nanoparticle carrier system was effective against neuroblastoma.

Nonetheless, a logical extension of our carrier system would be to show its usefulness against other aggressive cancers. Here we have shown that our lipophilic carrier enhanced the efficacy of GP and camptothecin against D54, an aggressive glioblastoma cell line (Figure 5). This would suggest that our lipophilic nanoparticle system is adaptable, and therefore promising as a new therapeutic approach against a range of cancers. Further work has indeed been planned in this direction.

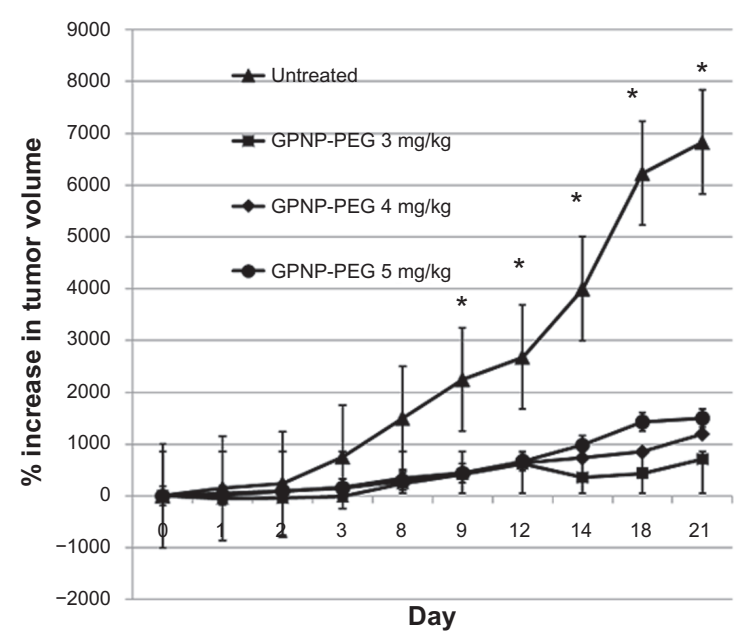

Figure 3 Tumor volume measurements after N2A-bearing A/J mice had received intraperitoneal injections of GPNP-PEG 3, 4, or $5 \mathrm{mg} / \mathrm{kg}$.

Notes: $\mathrm{n}=5-6, * P<0.05$.

Abbreviation: GPNP-PEG, gold porphyrin nanoparticles surface-coated with PEG.
A

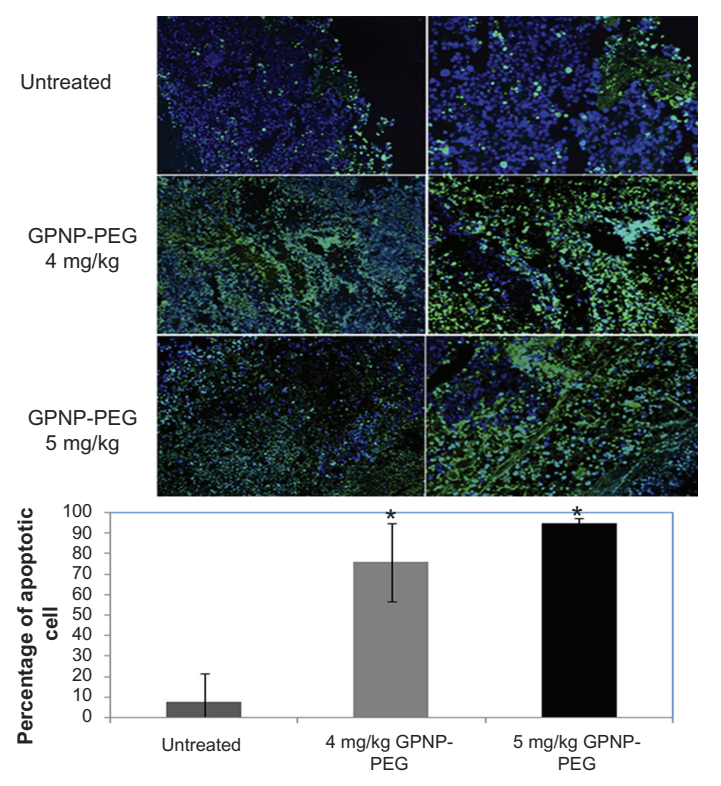

B

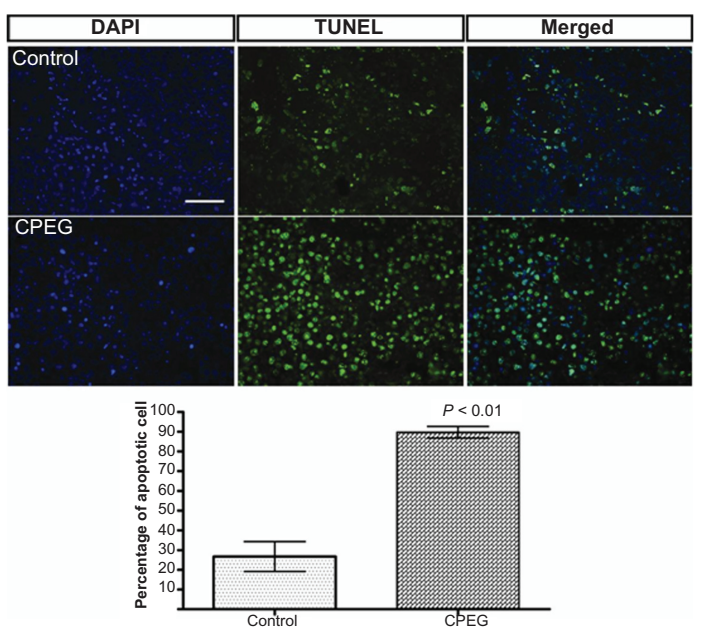

Figure 4 Apoptotic activity of (A) GP formulations and (B) camptothecin formulations using Tunel assay in neuroblastoma tissue harvested at 21 days after treatment. Notes: Green represents positive apoptotic cells and blue represents the DAPI nuclei stain. Magnification 100x (left column) or 200x (right column); $n=5, * P<0.05$ for $4 \mathrm{mg} / \mathrm{kg}$ or $5 \mathrm{mg} / \mathrm{kg}$ of GPNP-PEG in comparison with untreated controls in Figure 4A. Abbreviations: GP, gold porphyrin; GPNP-PEG, gold porphyrin nanoparticles surface-coated with PEG.

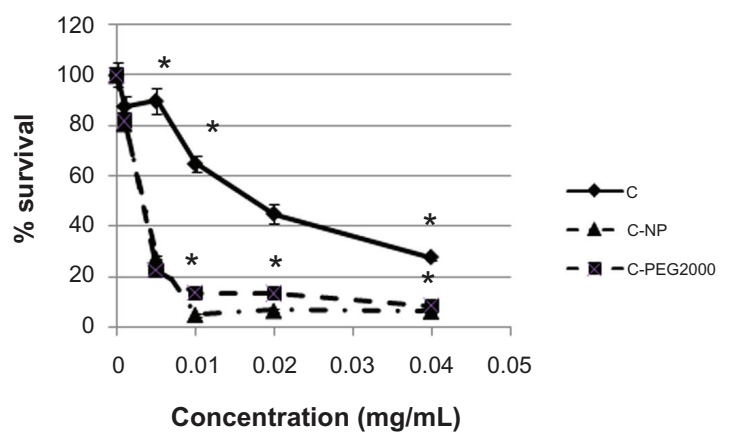

Figure 5 Dose-dependent antitumor activity against D54.

Notes: Results are presented as the mean \pm standard deviation $(n=4, * P<0.05$ for C-NP or C-PEG 2000 compared with C). 
To summarize, drug encapsulation into a PEGylated lipid nanoparticle carrier may further improve drug accumulation inside tumor tissue with low accumulation in the liver and provide effective antitumor activity in vitro and in vivo.

\section{Conclusion}

Our GPNP-PEG formulation offers a new strategy for the development of a safe and effective carrier for drug treatment of cancer. Clinical studies are eagerly awaited.

\section{Acknowledgment}

We thank Frankie Chan at the Electron Microscopy Unit in the Department of Pathology at Hong Kong University for providing assistance with electron microscopy. We also thank Timothy Tang in the Bioengineering Department at the University of Science and Technology in Hong Kong for providing technical assistance with the inductively coupled plasma-mass spectroscopy. This work was supported by the Hong Kong University Area of Excellence Scheme (AoE/P-10/01) and small project funding (grant 200907176012).

\section{Disclosure}

The authors report no conflicts of interest in this work.

\section{References}

1. Laverdiere C, Liu Q, Yasui Y, et al. Long-term outcomes in survivors of neuroblastoma: a report from the Childhood Cancer Survivor Study. $J$ Natl Cancer Inst. 2009;101:1131-1140.

2. To YF, Sun RW, Chen Y, et al. Gold (III) porphyrin complex is more potent than cisplatin in inhibiting growth of nasopharyngeal carcinoma in vitro and in vivo. Int J Cancer. 2009;124:1971-1979.

3. Lum CT, Yang ZF, Li HY, et al. Gold (III) compound is a novel chemocytotoxic agent for hepatocellular carcinoma. Int J Cancer. 2006;118: $1527-1538$

4. Li W, Xie Y, Sun RW, et al. Inhibition of Akt sensitizes neuroblastoma cells to gold (III) porphyrin 1a, a novel antitumour drug induced apoptosis and growth inhibition. Br J Cancer. 2009;101:342-349.

5. Sun RWY, Che CM. The anti-cancer properties of gold(III) compounds with dianionicporphyrin and tetradentate ligands. Coord Chem Rev. 2009; 253:1682-1691.

6. Pizzolato JF, Saltz LB. The camptothecins. Lancet. 2003;361: 2235-2242.

7. Sumitomo M, Koizumi F, Asano T, et al. Novel SN-38-incorporated polymeric micelle, NK012, strongly suppresses renal cancer progression. Cancer Res. 2008;68:1631-1635.

8. Boige V, Taieb J, Hebbar M, et al. Irinotecan as first-line chemotherapy in patients with advanced hepatocellular carcinoma: a multicenter phase II study with dose adjustment according to baseline serum bilirubin level. Eur J Cancer. 2006;42:456-459.

9. Romanelli S, Perego P, Graziella P, Cerenini N, Tortoreto M, Zunino F. In vitro and in vivo interaction between cisplatin and topotecan in ovarian carcinoma systems. Cancer Chemother Pharmacol. 1998;41: $385-390$.
10. Pantazis P, Kozielski AJ, Vardeman DM, Petry ER, Giovanella BC. Efficacy of camptothecin congeners in the treatment of human breast carcinoma xenografts. Oncol Res. 1994;5:273-281.

11. Liu Y, Zhang B, Yan B. Enabling anticancer therapeutics by nanoparticle carriers: the delivery of paclitaxel. Int J Mol Sci. 2011;12: 4395-4413.

12. Jun YJ, Jadhav VB, Min JH, et al. Stable and efficient delivery of docetaxel by micelle-encapsulation using a tripodalcyclotriphosphazene amphiphile. Int J Pharm. 2012;422:374-380.

13. Lu C, Liu P. Effect of chitosan multilayers encapsulation on controlled release performance of drug-loaded superparamagnetic alginate nanoparticles. J Mater Sci Mater Med. 2011. Epub Nov 5.

14. Trembley JH, Unger GM, Korman VL, et al. Nanoencapsulated antiCK2 small molecule drug or siRNA specifically targets malignant cancer but not benign cells. Cancer Lett. 2012;315:48-58.

15. Maeda $\mathrm{H}$. The enhanced permeability and retention (EPR) effect in tumor vasculature: the key role of tumor-selective macromolecular drug targeting. Adv Enzyme Regul. 2001;41:189-207.

16. Gaumet M, Vargas A, Gurny R, Delie F. Nanoparticles for drug delivery: the need for precision in reporting particle size parameters. Eur J Pharm Biopharm. 2008;69:1-9.

17. Storm G, Belliot SO, Daemen T, Lasic DD. Surface modification of nanoparticles to oppose uptake by the mononuclear phagocyte system. Adv Drug Deliv Rev. 1995;17:31-48.

18. Koziara JM, Oh JJ, Akers WS, Ferraris SP, Mumper RJ. Blood compatibility of cetyl alcohol/polysorbate-based nanoparticles. Pharm Res. 2005;22:1821-1828.

19. Lee PY, Zhu YZ, Yan JJ, et al. The cytotoxic effects of lipidic formulated gold-porphyrin nanoparticles for the treatment of neuroblastoma. Nanotechnol Sci Appl. 2010;3:23-28.

20. Che CM, Sun RWY, Yu WY, Ko CB, Zhu N, Sun H. Gold (III) porphyrins as a new class of anti-cancer drugs: cytotoxicity, DNA binding and induction of apoptosis in human cervix epitheloid cancer cells. Chem Commun (Camb). 2003;14:1718-1719.

21. Ma P, Dong X, Swadley CL, et al. Development of idarubicin and doxorubicin solid lipid nanoparticles to overcome pgp-mediated multiple drug resistance in leukemia. J Biomed Nanotechnol. 2009;5: 151-161.

22. Kumar VV, Chandrasekar D, Ramakrishna S, Kishan V, Rao YM, Diwan PV. Development and evaluation of nitrendipine loaded solid lipid nanoparticles: influence of wax and glyceride lipids on plasma pharmacokinetics. Int J Pharm. 2007;335:167-175.

23. De Cesare M, Calcaterra C, Pratesi G, et al. Eradication of ovarian tumor xenografts by locoregional administration of targeted immunotherapy. Clin Cancer Res. 2008;14:5512-5518.

24. Chlenski A, Guerrero LJ, Peddinti R, et al. Anti-angiogenic SPARC peptides inhibit progression of neuroblastoma tumors. Mol Cancer. 2010;9:138.

25. Shusterman S, Grupp SA, Barr R, Carpentieri D, Zhao H, Maris JM. The angiogenesis inhibitor tnp-470 effectively inhibits human neuroblastoma xenograft growth, especially in the setting of subclinical disease. Clin Cancer Res. 2001;7:977-984.

26. Phuangsab A, Lorence RM, Reichard KW, Peeples ME, Walter RJ. Newcastle disease virus therapy of human tumor xenografts: antitumor effects of local or systemic administration. Cancer Lett. 2001;172: $27-36$.

27. Kebbekus BB. Preparation of samples for metal analysis. In: Mitra S, editor. Sample Preparation Techniques in Analytical Chemistry. Hoboken, NJ: John Wiley \& Sons; 2003.

28. Drin G, Mazel M, Clair P, Mathieu D, Kaczorek M, Temsamani J. Physico-chemical requirements for cellular uptake of pAntp peptide. Role of lipid-binding affinity. Eur J Biochem. 2001;268: 1304-1314.

29. Braet F, Wisse E. Structural and functional aspects of liver sinusoidal endothelial cell fenestrae: a review. Comp Hepatol. 2002;1:1. 
30. Yuan F, Dellian M, Fukumura D, et al. Vascular permeability in a human tumor xenograft: molecular size dependence and cutoff size. Cancer Res. 1995;55:3752-3756.

31. Eisenbrey JR, Soulen MC, Wheatly MA. Delivery of encapsulated doxorubicin by ultrasound-mediated size reduction of drug-loaded polymer contrast agents. IEEE Trans Biomed Eng. 2010;57:24-28.
32. Fulda S. Tumor resistance to apoptosis. Int $J$ Cancer. 2009;124: 511-515.

International Journal of Nanomedicine

\section{Publish your work in this journal}

The International Journal of Nanomedicine is an international, peerreviewed journal focusing on the application of nanotechnology in diagnostics, therapeutics, and drug delivery systems throughout the biomedical field. This journal is indexed on PubMed Central,

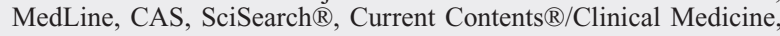

Journal Citation Reports/Science Edition, EMBase, Scopus and the Elsevier Bibliographic databases. The manuscript management system is completely online and includes a very quick and fair peer-review system, which is all easy to use. Visit http://www.dovepress.com/ testimonials.php to read real quotes from published authors.

Submit your manuscript here: http://www.dovepress.com/international-journal-of-nanomedicine-journal 